\title{
Manejo de infertilidad por anovulación en síndrome de ovario poliquístico
}

The manegement of anovulatory infertility in polycystic ovary syndrome

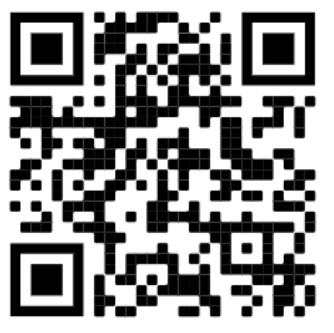

Recibido

$13 / 11 / 2020$

\author{
${ }^{1}$ Dra. Fiorella Otoya Chaves \\ Investigadora independiente, Heredia, Costa Rica \\ (i) https://orcid.org/0000-0002-2088-1154 \\ ${ }^{2}$ Dra. Stephanie León Quirós \\ Investigadora independiente, Heredia, Costa Rica \\ (D) https://orcid.org/0000-0002-4464-9501 \\ ${ }^{3}$ Dr. Mauricio Rodríguez Morera \\ Investigador independiente, San José, Costa Rica \\ (iD https://orcid.org/0000-0001-9453-7594
}

Corregido

$09 / 12 / 2020$
Aceptado

$11 / 01 / 2021$

\section{RESUMEN}

La infertilidad por anovulación es una complicación común asociada al síndrome de ovario poliquístico. La optimización de la salud constituye el primer paso en el abordaje de esta patología, principalmente con cambios en estilo de vida e índice de masa corporal óptimo. En lo referente a farmacoterapia, el citrato de clomifeno es ampliamente utilizado a nivel mundial como primera línea para inducir la ovulación. No obstante, el letrozol y la metformina en combinación con citrato de clomifeno, son terapias con evidencia prometedora que han demostrado ser superiores al citrato de clomifeno en monoterapia, en lo que respecta a fertilidad. Sin embargo, no existe consenso, y se siguen considerando terapias de segunda línea. Las gonadotropinas exógenas y la perforación laparoscópica del ovario son utilizadas cuando hay resistencia a la primera línea. La fertilización in vitro es una opción cuando tanto la primera, como la segunda línea, no son exitosas. Otros medicamentos se encuentran en estudio para determinar su eficacia en esta patología, como los agonistas del receptor del péptido similar al glucagón tipo 1 y esteroisómeros del inositol, empero, aún requieren estudios de mejor calidad.

PALABRAS CLAVE: anovulación; infertilidad; síndrome de ovario poliquístico; clomifeno, letrozol.

\footnotetext{
ABSTRACT

Anovulatory infertility is a common complication associated with polycystic ovary syndrome. Health optimization is the first step in the management of this pathology, specially focused on lifestyle changes and optimal body mass index. About pharmacotherapy, clomiphene citrate is used worldwide as a first

1 Médica general, graduada de la Universidad de Costa Rica (UCR), cod. MED16804, correo: fiorella.otoya.chaves@gmail.com

${ }^{2}$ Médica general, graduada de la Universidad de Costa Rica (UCR), cod. MED16835, correo: spleon12@hotmail.com

${ }^{3}$ Médico general, graduado de la Universidad de Costa Rica (UCR), cod. MED16823, correo: mauricio.rodríguez.morera@gmail.com
} 
line treatment to induce ovulation. However, letrozol and a combination of clomiphene citrate plus metformin have shown to be superior to clomiphene citrate in monotherapy, concerning fertility. These are still considered second line treatment due to lack of consensus. Exogenous gonadotropins and laparoscopic ovarian drilling are used when first-line treatment resistance is determined. Once first- and second-line treatment have failed, in-vitro fertilization is an option. Other drugs are being studied to determine their efficacy on this pathology, such as glucagon-like peptide 1 receptor agonists and inositol stereoisomers, but higher quality studies are still required.

KEYWORDS: anovulation; infertility; polycystic ovary syndrome; clomiphene; letrozole.

\section{INTRODUCCIÓN}

El síndrome de ovario poliquístico (SOPQ) se considera el trastorno endocrino más común entre las mujeres en edad reproductiva. Se observa entre el $4 \%$ al $18 \%$ de estas personas. Se cree que los desórdenes relacionados a la ovulación ocupan un $25-50 \%$ de las causas de infertilidad femenina, y el SOPQ es considerado una de las principales causas de anovulación, por ende, causa importante de infertilidad (1-4). La anovulación en sopq es producto de una alteración en el eje hipotálamo-hipófisis-ovario. Se presenta también hiperinsulinemia, la cual afecta el desarrollo normal de los folículos ováricos y genera un aumento en los niveles de andrógenos, estrógenos y de globulina ligadora de hormonas sexuales, y otros mecanismos aún no bien establecidos $(2,3)$. Para el adecuado manejo, se busca primero optimizar la salud de la paciente. Esto mediante una mejoría del índice de masa corporal, debido a la influencia del sobrepeso u obesidad en la anovulación. Una vez que logrados estos cambios, se opta por tratamientos de primera o segunda línea para así lograr la inducción de la ovulación. No obstante, si estas fallan o existe otro componente asociado a la subfertilidad presentada, existe la opción de terapias de reproducción asistida (1). Este artículo pretende revisar el tratamiento actualizado que forma parte del abordaje de pacientes con infertilidad a causa de anovulación por SOPQ.

\section{MÉTODO}

Se realizó una búsqueda de artículos científicos, con prioridad a aquellos publicados del 2015 al 2020, se utilizaron las palabras clave "Síndrome de ovario poliquístico", "infertilidad", "citrato de clomifeno", "anovulación" y "letrozol", en las siguientes bases de datos: ScienceDirect, PubMed, Cochrane, SpringerLink, Sistema de Bibliotecas Documentación e Información (SIBDI) de la Universidad de Costa Rica, la Biblioteca Nacional de Salud y Seguridad Social (BINASSS) y SciELO. Se excluyeron aquellos escritos publicados en idiomas diferentes al español o inglés, artículos no referidos al tópico, artículos publicados antes del año 2010 o aquellos que no contaran con el artículo en texto completo pese a cumplir criterios de inclusión. Finalmente, se incluyeron en esta revisión, 31 artículos publicados del 2015 al 2020, y 5 artículos publicados del 2010 al 2015, que cumplieron con los requisitos antes descritos.

\section{ETIOLOGÍA}

En el caso del SOPQ, la disfunción ovárica se ha visto relacionada con una alteración en 
el eje hipotálamo-hipófisis-ovario. Existe en esta patología un aumento en la frecuencia de los pulsos de hormona liberadora de gonadotropinas $(\mathrm{GnRH}$, por sus siglas en inglés), aumento en los niveles de hormona luteinizante y aumento en los niveles de kisspeptina, todos siendo factores que se favorecen la anovulación (5).

La resistencia a la insulina se observa a menudo en pacientes con SOPQ y puede llevar a hiperinsulinemia, esto afecta el desarrollo normal de los folículos ováricos y se genera un aumento en los niveles tanto de andrógenos como de estrógenos. Además, hay disminución en las concentraciones de globulina ligadora de hormonas sexuales (SHBG, por sus siglas en inglés) que, sumado al aumento de la hormona luteinizante, evitan la ovulación. Aunado a esto, la elevación en niveles de andrógenos circulantes en ausencia de ovulación puede llegar a asociar hiperplasia endometrial, resultando también en infertilidad (6). También, se ha observado la importancia de la elevación en los niveles de hormona anti-Mülleriana $(\mathrm{AMH}$, por sus siglas en inglés) en estas pacientes que padecen infertilidad, en ellas se encuentran valores de 2 a 3 veces mayores de $\mathrm{AMH}$ que en aquellas, por lo demás, sanas. La capacidad de la $\mathrm{AMH}$ de regular de forma negativa los folículos principales es lo que le da un papel significante en la subfertilidad que es consecuencia del SOPQ (7).

\section{ABORDAJE ACTUAL PARA INFERTILIDAD}

Previo a iniciar fármacos en las pacientes para tratar su subfertilidad, se debe buscar optimizar su salud. Se recomienda lograr en la paciente una mejoría o normalización en el índice de masa corporal (IMC). Se debe explicar a la mujer la importancia del control de su peso y cómo este está influyendo en la subfertilidad que padece. Posteriormente se cuenta con terapias farmacológicas aprobadas tanto para primera y segunda línea, cuando estas han fallado en inducir la ovulación, o si se ha llegado a identificar otro factor asociado a la subfertilidad que padece la paciente, puede optarse por la fertilización in-vitro (IVF, por sus siglas en inglés) $(5,8)$.

\section{$\underline{\text { Intervenciones no farmacológicas }}$}

Este es un factor determinante al abordar a una paciente que padece infertilidad por $S O P Q$, ya que se ha visto que estas mujeres tienden a presentar menores tasas de éxito que otras, no obesas, con este mismo diagnóstico. Este exceso de peso conlleva a anomalías tanto endocrinas como metabólicas que finalmente alteran el funcionamiento normal de diversos órganos, incluyendo los ovarios y la función reproductiva tanto en hombres como en mujeres (9). Se ha sugerido que con el aumento en la grasa corporal y la disfunción del tejido adiposo que resulta del SOPQ, aumenta la producción de resistina y leptina, y la concentración de adiponectina disminuye. Esta última tiende a tener un comportamiento asociado al IMC de la persona, donde disminuye cuando existe obesidad y se eleva con la pérdida de peso. Además, la leptina se secreta en proporción con la masa adiposa (10).

Dentro de los efectos de la adiponectina, en relación con la infertilidad, se ha planteado que puede tener acción en la producción de gonadotropinas a nivel del sistema nervioso central y en el ovario regula factores asociados a la ovulación (11).

Por lo anterior, aunque un IMC normal sería ideal, una pérdida de peso del $5-10 \%$ es beneficiosa, con esta perdida de peso corporal, se logren mejores resultados, que 
en pacientes con los mismos padecimientos pero que persisten con obesidad o no disminuyen su peso $(5,12)$.

\section{$\underline{\text { Tatamiento de primera línea }}$}

Los fármacos orales inductores de la ovulación ocupan el primer lugar en la lista de tratamientos disponibles. Dentro de esta categoría de fármacos, los más utilizados son clomifeno y letrozol $(1,5)$.

- Citrato de clomifeno: el citrato de clomifeno (CC) es un fármaco antagonista competitivo de estrógeno, que al unirse y, por ende, bloquear los receptores nucleares de esta hormona a nivel hipotalámico, evita que se interpreten correctamente los niveles de estrógeno circulantes, por lo que de forma compensatoria aumentan los pulsos de hormona liberadora de gonadotropinas. Con esto, se envía una señal a la hipófisis para que se dé la liberación de gonadotropinas, este estímulo causa un aumento en la secreción de hormona folículo estimulante y genera la ovulación $(1,2,5)$. Este es el único fármaco inductor de la ovulación aprobado por la Administración de Medicamentos y Alimentos de Estados Unidos (FDA, por sus siglas en inglés), y ha demostrado alto porcentaje de éxito en diversos estudios. Sin embargo, es importante que el clínico tome en cuenta que, paradójicamente, esta droga puede dificultar la concepción e implantación del cigoto, ya que su efecto no se limita a los ovarios, sino que puede afectar otros órganos. Un endometrio delgado y moco cervical espeso son efectos del CC en el útero que pueden jugar un papel a favor de la subfertilidad en un subgrupo de mujeres, aún después de lograr la ovulación $(2,5)$. Respecto a sus efectos adversos, se reconocen los asociados a un estado hipoestrogénico, alteraciones visuales, mareos, además de la posibilidad de inducir el desarrollo multifolicular que, hasta en un $11 \%$, puede tener como consecuencia embarazos múltiples y en un porcentaje aún menor puede culminar en un síndrome de hiperestimulación ovárica (OHSS, por sus siglas en inglés). Estas pacientes se benefician de un adecuado monitoreo, el cual debe ser frecuente, con lo que se podrá suspender el tratamiento si se detectan signos de una respuesta excesiva y así evitar estas complicaciones $(2,5,8)$.

- Letrozol: inhibidor de aromatasa que actúa mediante dos mecanismos, inhibiendo la conversión de androstenediona a estrona y testosterona a estradiol, además, previene la biosíntesis de estrógenos a nivel sistémico. Es así como se logra generar una retroalimentación hacia el eje hipotálamo-hipófisis que resulta en un aumento de las gonadotropinas y por ende, en la inducción de la ovulación. Adicionalmente, este fármaco no tiene efecto en el endometrio ni en moco cervical (13). El riesgo de desarrollo multifolicular en este caso es menor, pues el sistema de retroalimentación entre el hipotálamo y la hipófisis está intacto, a diferencia de lo que sucede con el CC, donde la inhibición se da en los receptores de estrógeno hipotalámicos (5).

- Citrato de clomifeno versus letrozol: el citrato de clomifeno fue por muchos 
años el medicamento de primera línea para tratar la anovulación inducida por SOPQ que implica subfertilidad. Sin embargo, en este momento se cuenta con evidencia actualizada que apunta al letrozol como posible fármaco de primera línea (5).

Parece ser que esta droga tiene mayor éxito en lograr embarazos con nacidos vivos que el CC, esta conclusión está basada en ensayos controlados aleatorizados con 2954 sujetos de estudio. Además, existe evidencia calificada de alta calidad que indica que no existen diferencias significativas para el riesgo de abortos espontáneos, embarazos múltiples ni teratogenicidad, entre ambas drogas. Aunado a esto, la vida media del letrozol es menor, por lo que se espera que se haya aclarado del cuerpo previo a la implantación evitando los efectos observados en el moco cervical y el endometrio con el CC (14).

Actualmente, algunos autores recomiendan el letrozol como fármaco de primera línea, y no el CC, a menos de que no se encuentre disponible o no se tenga la capacidad de cubrir su costo económico. Mientras que otros autores aún consideran insuficiente la evidencia, y discuten considerarlo como segunda línea de tratamiento farmacológico, cuando se haya presentado resistencia al CC (5).

Un metanálisis de diciembre publicado en el 2017 estudió 8 tratamientos para inducir la ovulación en mujeres con SOPQ, incluyendo 57 estudios y un total de 8082 mujeres. Dentro de sus conclusiones indican que tanto el letrozol, como el CC más metformina tienen mejores resultados que el $\mathrm{CC}$ en monoterapia, en términos de ovulación y embarazo. Por lo que, sustenta las recomendaciones de letrozol como posible primera línea de tratamiento (15).

\section{Tratamiento de segunda línea}

Este grupo de terapias son parte de las opciones que actualmente se presentan a las pacientes una vez que se ha diagnosticado resistencia al CC. Dentro de estas terapias las utilizadas son, la metformina, gonadotropinas y la perforación ovárica laparoscópica $(16,17)$.

- Metformina combinada con citrato de clomifeno: este es un fármaco derivado sintético de biguanida que se utiliza en combinación con el CC en las pacientes resistentes a esta última droga en monoterapia. Aunque se ha estudiado el papel de la metformina en monoterapia, y pese a elevar las tasas de ovulación, no logra superar la eficacia del citrato de clomifeno, el letrozol o la terapia combinada de CC con metformina. Empero, la terapia combinada con CC sí cuenta con evidencia que indica que podría considerarse como terapia de primera línea $(15,18,19)$.

Respecto a su mecanismo de acción, se debe mencionar el papel de la $\mathrm{AMH}$ en la subfertilidad que se observa en pacientes con SOPQ, ya que esta regula de forma negativa los folículos principales. Las células de la granulosa de los folículos pre-antrales y antrales pequeños son las encargadas principales de la secreción de la $\mathrm{AMH}$. Se ha documentado que las pacientes con SOPQ pueden tener valores de 2 a 3 veces mayores que otras mujeres sanas (7).

La metformina logra disminuir los niveles de $\mathrm{AMH}$, y esto se presume que está asociado a un mecanismo insulino- 
dependiente. La hiperinsulinemia se ha relacionado con la elevación de esta hormona, y además se asocia con anovulación e hiperandrogenismo. Se sugiere que la disminución en la concentración de AMH se logra observar a las 8 semanas de iniciado el tratamiento y puede ser dependiente del IMC de la persona; a mayor IMC, mayor disminución de dichos niveles (7).

También, la metformina logra aumentar la producción de SHBG y disminuir la circulación de andrógenos, con lo que mejora el funcionamiento de las células de la teca y la granulosa. Asimismo, las neuronas encargadas de liberar $\mathrm{GnRH}$ regulan la fertilidad con un mecanismo dependiente de proteína quinasa activada por AMP (AMPK, por sus siglas en inglés), que es inhibida por este fármaco. Con ambos mecanismos anteriores, también se podría lograr mejorar la tasa de fertilidad en estas mujeres gracias al uso de metformina. Existe también evidencia de un posible mecanismo mediado por la inhibición del complejo I mitocondrial que podría inhibir directamente la esteroidogénesis a nivel del ovario $(6,20)$.

Además de su papel para lograr el embarazo, se han reportado también beneficios en continuar el tratamiento a lo largo del embarazo en mujeres con diagnóstico de SOPQ. Un metanálisis del 2016 que incluyó 13 estudios y 1606 mujeres embarazadas con SOPQ, concluyó que el uso de metformina durante este periodo, podría reducir las tasas de abortos espontáneos, parto prematuro, y aumentar las posibilidades de lograr un parto de término. Además, logra reducir la incidencia de diabetes gestacional e hipertensión relacionada al embarazo (21).
- Gonadotropinas: son hormonas exógenas utilizadas una vez que los cambios en el estilo de vida y los tratamientos orales no han sido exitosos en lograr la ovulación o embarazo. Estas hormonas se derivan de extractos urinarios 0 mediante tecnología recombinante. Con la hormona folículo estimulante (FSH, por sus siglas en inglés) exógena se busca estimular la proliferación de las células de la granulosa y el crecimiento folicular, y con la hormona luteinizante ( $\mathrm{LH}$, por sus siglas en inglés) se logra la producción de estradiol, ya que se secretan andrógenos que serán aromatizados posteriormente. Con esto se desea promover el desarrollo de un único folículo maduro (22).

Respecto a las gonadotropinas urinarias versus las recombinantes, parece existir poca o nula diferencia en tasas de embarazo, nacidos vivos, embarazos múltiples o pérdidas. Por lo que, de acuerdo con la evidencia actual, no existe ventaja significativa entre las preparaciones utilizadas de gonadotropinas respecto a la tasa de embarazos logrados $(22,23)$.

Según concluyen Wang et al, en su metanálisis, las gonadotropinas representan una opción efectiva de tratamiento para lograr la ovulación, sin embargo, las probabilidades de embarazos múltiples son mayores que las registradas con letrozol, citrato de clomifeno y metformina. Por esto se consideran como terapia de segunda línea y no se recomiendan como un adecuado abordaje medicamentoso inicial. Sin embargo, otro estudio que comparó el citrato de clomifeno en monoterapia con las gonadotropinas, reportó mayores tasas de embarazos 
con nacidos vivos sin aumentar la incidencia de embarazos múltiples, pero sí con mayores tasas de abortos espontáneos $(15,23)$.

Su seguridad se puso en duda ante la preocupación de aumento de aneuploidías tras el uso de gonadotropinas para inducir la ovulación. Sin embargo, un estudio prospectivo observacional publicado en el 2019 indica que las tasas de aneuploidía embrionaria no aumentan con el uso de esta droga (24).

- Cirugía laparoscópica: es un procedimiento quirúrgico llamado perforación ovárica laparoscópica (LOD, por sus siglas en inglés). Se cree que la destrucción local de los folículos y el estroma ovárico produce una caída en los niveles de andrógenos e inhibina séricos y locales, por lo que aumenta la FSH permitiendo el desarrollo folicular $(25,26)$.

Según una revisión de Cochrane del 2019, que incluyó 38 ensayos con un total de 3326 mujeres, concluyó con evidencia de moderada calidad que este procedimiento reduce el número de embarazos múltiples. Además, con evidencia de baja calidad indica que comparado con otros abordajes, la probabilidad de lograr un embarazo no es diferente entre tratamientos. No se obtuvo información respecto a las tasas de abortos espontáneos, comparando LOD con medicamentos para inducir la ovulación (25).

En la actualidad se cuenta con la opción de cirugía unilateral (ULOD) o bilateral (BLOD). En torno a ambas técnicas se han realizado estudios para determinar cuál es el mejor abordaje, con mejores tasas de embarazos y menores efectos adversos. Un estudio del 2017 que incluyó 8 artículos con 484 participantes de las cuales 240 mujeres recibieron el tratamiento con ULOD y 244 con BLOD. Se concluyó, con evidencia de baja a moderada calidad, que no existe diferencia significativa respecto a nacidos vivos ni abortos espontáneos. Sí se encontraron diferencias respecto a concentraciones de $\mathrm{AMH}$ y el conteo de folículos antrales (AFC, según sus siglas en inglés). En el caso de $\mathrm{AMH}$ es mayor la reducción en las pacientes que recibieron ULOD, asimismo en este grupo de pacientes se observó un mayor AFC, ambos resultados reportados en el seguimiento a los 6 meses (26).

\section{Terapias complementarias y alternativas} $\underline{\text { en estudio }}$

- Fertilización in vitro: el número de pacientes con SOPQ que están sometiéndose a terapias de reproducción asistida ha incrementado a lo largo del tiempo. La fertilización in vitro convencional y la técnica con inyección citoplasmática de esperma (ICSI, por sus siglas en inglés) son las principales terapias utilizadas. En la técnica de ICSI el proceso de fertilización se lleva a cabo, principalmente, mediante maduración in vitro (IVM, por sus siglas en inglés) $(27,28)$.

Al comparar ambas técnicas, se tiene que la IVF convencional logra tasas de embarazo, implantación y, número y calidad de embriones mayores a las que se obtienen con IVM. Sin embargo, la IVM tiene la ventaja de evitar la hiperestimulación ovárica, tiene un costo más bajo y logra tasas de embarazo aceptables, aunque menores a la técnica convencional $(27,28)$. 
Respecto a la problemática de la hiperestimulación ovárica y embarazos múltiples, actualmente se toma en cuenta el número de folículos, los niveles de estradiol sérico y la cantidad de ovocitos reclutados, para intentar prevenir la respuesta exagerada en los ovarios. Aunque se debe tener claro que este síndrome puede ser difícil de predecir y su severidad o necesidad de hospitalización deben ser analizadas de forma individual con cada paciente (29).

Por otra parte, el uso de metformina asociado a esta técnica de reproducción ha reportado mejoría en el éxito de la IVF, así como mejoría de los síntomas del SOPQ, y además, no asocia daños teratogénicos. También, la obesidad se ha relacionado con mayores tasas de abortos espontáneos y menores tasas de implantación, embarazo clínico, y partos. Por lo que se recomienda fomentar la pérdida de peso en pacientes obesas que desean optar por esta técnica de reproducción asistida (30-32).

- Agonista del receptor de péptido similar al glucagón tipo 1 (GLP-1): actualmente no se conoce el mecanismo por el cual la capacidad de reproducción femenina se beneficia del uso de estos fármacos, donde el más utilizado es el liraglutide. Algunos de los beneficios descritos son, ciclos menstruales regulares, aumento en la SHBG y una disminución en los niveles de testosterona y el volumen de los ovarios $(32,33)$.

Un estudio del 2019, con evidencia de baja a moderada calidad, demostró que al compararse con metformina, los agonistas del receptor de GLP-1 son superiores en mejorar la sensibilidad a la insulina. Se ha descrito también una terapia combinada con metformina, con la que se logra mayor pérdida de peso que con la metformina en monoterapia. Esto se ve relacionado a un mecanismo que involucra el efecto del liraglutide a nivel hipotalámico, que produce una reducción en la ingesta de alimentos (32,34).

Aún se requieren más estudios, con evidencia de mejor calidad, para determinar el papel del liraglutide $u$ otros fármacos de la misma familia. Aunque la evidencia clínica y experimental sugiere que sí existe una asociación entre la obesidad, las alteraciones del GLP-1 y la patogénesis del SOPQ (33).

- Mioinositol en combinación con Dchiro-inositol: el mioinositol y el Dchiro-inositol son esteroisómeros del inositol, y ambos funcionan como mediadores de la acción de la insulina en la célula (35).

En un estudio del año 2017 donde se utilizaron estas drogas en combinación como tratamiento de primera línea en pacientes con SOPQ, se observó que mejoran las concentraciones séricas de andrógenos, también mejora la proporción de LH-FSH y se regulan los ciclos menstruales. Se logró contribuir a un aumento de la fertilidad, aunque aún se requieren más estudios con estos fármacos (35).

Según una revisión de Cochrane del 2018, con 13 ensayos y 1472 pacientes, donde se buscaba la existencia de beneficios al tomar suplementos de inositol en pacientes que se sometían a IVF. Concluyeron que la evidencia es de muy baja calidad. No se obtuvo datos significativos respecto a diferencias en tasas de nacidos vivos, embarazos clínicos, tasas de aborto espontáneo o embarazo múltiple; al compararlo con la terapia convencional utilizada en IVF. 
Por lo que respalda la necesidad de nuevos y mejores estudios (36).

\section{CONCLUSIÓN}

De acuerdo con la información disponible actualmente respecto a la primera línea de tratamiento, el letrozol y la combinación de CC con metformina, podrían ser la mejor opción, ya que se cuenta con evidencia que indica que estos abordajes logran mayores tasas de ovulación y embarazo, con menor riesgo de una respuesta exagerada y por ende, menores tasas de embarazos múltiples y OHSS. De ambas alternativas, el letrozol es el único con el que se han demostrado mayores tasas de nacidos vivos, en comparación con el CC en monoterapia.

Sin embargo, pese a la prometedora evidencia, ninguno ha sido aprobado para reemplazar al CC en monoterapia como el tratamiento de primera línea en muchos países y su uso se limita a segunda línea, una vez que se haya presentado resistencia al CC. Los tratamientos de segunda línea con gonadotropinas exógenas y LOD, son eficaces en lograr ovulación y embarazos con nacidos vivos en tasas importantes. Sin embargo, las mayores tasas de embarazos múltiples y respuesta ovárica exagerada, en el caso de las gonadotropinas exógenas, y la naturaleza quirúrgica de la LOD, las mantiene como opciones de segunda línea. La IVF se contempla una vez que no se ha tenido éxito con las terapias de primera y segunda línea. Tanto la IVF convencional como la ICSI cuentan con tasas de embarazo aceptables, aunque mayores en el caso de la terapia convencional. Además, con la ICSI se evita la hiperestimulación ovárica y embarazos múltiples.

Por último, el mioinositol y los agonistas del receptor de GLP-1, son alternativas potenciales para el abordaje de estas pacientes, pero aún requieren más estudios con evidencia de mejor calidad.

Las terapias antes descritas, tienen mayores tasas de éxito cuando, previamente, se ha logrado normalizar el IMC o al menos, lograr una reducción de peso de 5 a 10\%.

\section{REFERENCIAS}

1. Wang R, Li W, Bordewijk EM, Legro RS, Zhang H, Wu X, et al. First-line ovula- tion induction for polycystic ovary syndrome: an individual participant data meta-analysis. Hum Reprod Update. 2019;25:71732. https://doi.org/10.1093/humupd/dmz029

2. Bergh C, Moore M, Gundell C. Evidence-Based Management of Infertility in Women With Polycystic Ovary Syndrome. JOGNN. 2016;45:111-122. http://dx.doi.org/10.1016/j.jogn.2015.10.001

3. Zhang J, Si Q, Li J. Therapeutic effects of metformin and clomiphene in combination with lifestyle intervention on infertility in women with obese polycystic ovary syndrome. Pak J Med Sci. 2017;33(1):8-12. doi: https://doi.org/10.12669/pjms.331.11764

4. Giviziez C, Sanchez E, Approbato M, Maia M, Fleury E et al. Obesity and anovulatory infertility: A review. JBRA Assisted Reproduction. 2016;20(4):240-245. https://doi.org/10.5935/1518-0557.20160046

5. Mascarenhas M, Balen A. Treatment update for anovulation and subfertility in polycystic ovary syndrome. Current Opinion in Endocrine and Metabolic Research. 2020;12:53-58. https://doi.org/10.1016/j.coemr.2020.03.003

6. Khan A, Karim N, Ainuddin J. The Triad Of PCOS, Infertility And Metformin. JBUMDC. 2019;9(1):67-71. 
7. Foroozanfard F, Samimi M, Almadani K, Sehat M. Effect of metformin on the anti-Müllerian hormone level in infertile women with polycystic ovarian syndrome. Electronic Physician. 2017;9(12):5969-5973. https://doi.org/10.19082/5969

8. Balen A, Morley L, Misso M, Franks S, Legro R, et al. The management of anovulatory infertility in women with polycystic ovary syndrome: an analysis of the evidence to support the development of global WHO guidance. Hum Reprod Update. 2016;22(6):687-708. https://doi.org/10.1093/humupd/dmw025

9. Sánchez C. Factores que influyen en la tasa de embarazo en mujeres obesas en tratamiento con inseminación artificial. NURE Inv. 2013;10(62).

10. Obirikorang C, Owiredu W, Adu-Afram S, Acheampong E, Asamoah E,et al. Assessing the variability and predictability of adipokines (adiponectin, leptin, resistin and their ratios) in non-obese and obese women with anovulatory polycystic ovary syndrome. BMC Res Notes. 2019;12:513. https://doi.org/10.1186/s13104-019-4546z.

11. Vivas C, Calderón C, Pulido E. Adiponectina y Reproducción. Rev Desafíos. 2013;1(7). Accesado el 24 de setiembre 2020, en: http://revistas.ut.edu.co/index.php/desafios/article/view/439

12. Fichman V, Costa RS, Miglioli TC, Marinheiro LP. Association of obesity and anovulatory infertility. Einstein (São Paulo). 2020;18:eAO5150. http://dx.doi.org/10.31744/einstein journal/2020AO5150

13. Kar S. Clomiphene citrate or letrozole as first-line ovulation induction drug in infertile PCOS women: a prospective randomized trial. J Hum Reprod Sci. 2012;5:262-265. https://doi.org/10.4103/0974-1208.106338

14. Franik S, Eltrop SM, Kremer JAM, Kiesel L, Farquhar C. Aromatase inhibitors (letrozole) for subfertile women with polycystic ovary syndrome.TheCochrane database of systematic reviews.2018;5(5). https://doi.org/10.1002/14651858.CD010287.pub3

15. Wang R, Kim BV, van Wely M, Johnson NP, Costello M, et al. Treatment strategies for women with WHO group II anovulation: systematic review and network meta-analysis. BMJ. 2017;356:j138. https://doi.org/10.1136/bmj.j138

16. Debras E, Fernandez H, Neveu ME, Deffieux X, Capmas P. Ovarian drilling in polycystic ovary syndrome: Long term pregnancy rate. Eur J Obstet Gynecol Reprod Biol X. 2019;4:100093. https://doi.org/10.1016/j.eurox.2019.100093

17. Yu Q, Hu S, Wang Y, Cheng G, Xia W, et al. Letrozole versus laparoscopic ovarian drilling in clomiphene citrateresistant women with polycystic ovary syndrome: a systematic review and meta-analysis of randomized controlled trials. Reprod Biol Endocrinol. 2019;17:17. https://doi.org/10.1186/s12958-019-0461-3

18. Costello M, Garad R, Hart R, Homer H, Johnson L, et al. A Review of Second- and Third-line Infertility Treatments and Supporting Evidence in Women with Polycystic Ovary Syndrome. Med Sci. 2019;7,75. https://doi.org/10.3390/medsci7070075

19. Practice Committee of the American Society for Reproductive Medicine. Role of metformin for ovulation induction in infertile patients with polycystic ovary syndrome (PCOS): a guideline. Fertil Steril. 2017;108:426-441. https://doi.org/10.1016/j.fertnstert.2017.06.026

20. Sam S, Ehrmann D. Metformin therapy for the reproductive and metabolic consequences of polycystic ovary syndrome. Diabetologia. 2017;60:1656-1661. https://doi.org/10.1007/s00125-017-4306-3

21. Zeng XL, Zhang YF, Quan T, Xue Y, An RF. Effects of metformin on pregnancy outcomes in women with polycystic ovary syndrome. Medicine. 2016;95(36). https://doi.org/10.1097/MD.0000000000004526

e642 Revista Médica Sinergia Vol.6 (2), Febrero 2021 - ISSN:2215-4523 / e-ISSN:2215-5279 
22. Practice Committee of the American Society for Reproductive Medicine. Use of exogenous gonadotropins for ovulation induction in anovulatory women: a committe opinion. Fertil Steril. 2020;113(1):66-69. https://doi.org/10.1016/j.fertnstert.2019.09.020

23. Weiss NS, Kostova E, Nahuis M, Mol BWJ, van der Veen F, et al.Gonadotrophins for ovulation induction in women with polycystic ovary syndrome. Cochrane Database of Systematic Reviews. 2019;1:CD010290. https://doi.org/10.1002/14651858.CD010290.pub3

24. Hong K, Franasiak J, Werner M, Patounakis G, Juneau C, et al. Embryonic aneuploidy rates are equivalent in natural cycles and gonadotropin-stimulated cycles. Fertil Steril. 2020;112(4):670-676. https://doi.org/10.1016/j.fertnstert.2019.05.039

25. Bordewijk EM, Ng KYB, Rakic L, Mol BWJ, Brown J, Crawford TJ, et al. Laparoscopic ovarian drilling for ovulation induction in women with anovulatory polycystic ovary syndrome. Cochrane Database of Systematic Reviews. 2020;2:CD001122. https://doi.org/10.1002/14651858.CD001122.pub5

26. Abu Hatem, Foda O, Rakhawy M. Unilateral or bilateral laparoscopic ovarian drilling in polycystic ovary syndrome: a meta analysis of randomized trials. Arch Gynecol Obstet. 2018;297:859-870. https://doi.org/10.1007/s00404$\underline{018-4680-1}$

27. Elshewy N, Ji D, Zhang Z, Chen D, Chen B, et al. Association between mild stimulated IVF/M cycle and early embryo arrest in sub fertile women with/without PCOS. Reprod Biol Endocrinol. 2020;18:71. https://doi.org/10.1186/s12958-020-00622-y

28. Walls M, Junk S, Ryan JP, Hart R. IVF versus ICSI for the fertilization of in-vitro matured human oocytes. rBMO. 2012;25:603-607.https://doi.org/10.1016/j.rbmo.2012.08.001

29. 29. Swanton A, Storey L, McVeigh E, Child T. IVF outcome in women with PCOS, PCO and normal ovarian $\begin{array}{lllll}\text { morphology.Eur J Obstet Gynecol Reprod } & \text { Biol. 2010;149:68-71 }\end{array}$ https://doi.org/10.1016/j.ejogrb.2009.11.017

30. Mateo HA, Rentería GA, Frausto JA, Mateo M y col. Administración de metformina en mujeres con síndrome de ovario poliquístico y su efecto en fertilización in vitro. Reproducción (México). 2018;9(2):31-37.

31. Zhou H, Zhang D, Luo Z, Yang A, Cui N, et al. Association between Body Mass Index and Reproductive Outcome in Women with Polycystic Ovary Syndrome Receiving IVF/ICSI-ET. BioMed Research International. 2020;2020:6434080. https://doi.org/10.1155/2020/6434080

32. Salamun V, Jensterle M, Janez A, Bokal E. Liraglutide increases IVF pregnancy rates in obese PCOS women with poor response to first-line reproductive treatments: a pilot randomized study. Eur J Endocrinol. 2018;179(1):1-11. https://doi.org/10.1530/EJE-18-0175

33. Cena H, Chiovato L, Nappi Rossella. Obesity, Polycystic Ovary Syndrome, and Infertility: A New Avenue for GLP1 Receptor Agonists. Clin Endocrinol Metab. 2020;105(8):e2695-e2709. https://doi.org/10.1210/clinem/dgaa285

34. Han Y, Li Y, He B. GLP-1 receptor agonists versus metformin in PCOS: a systematic review and meta-analysis. rBMO. 2019;39(2):332-342. https://doi.org/10.1016/..rbmo.2019.04.017

35. Carmona IO, Saucedo E, Moraga MR, Romeu A. Mioinositol en combinación con D-chiro-inositol: resultados preliminares en el tratamiento de primera línea de pacientes con síndrome de ovario poliquístico. Ginecol Obstet Mex. 2017;85(3):141-151.

e642 Revista Médica Sinergia Vol.6 (2), Febrero 2021 - ISSN:2215-4523 / e-ISSN:2215-5279 
36. Showell MG, Mackenzie-Proctor R, Jordan V, Hodgson R, Farquhar C. Inositol for subfertile women with polycystic ovary syndrome. Cochrane Database of Systematic Reviews. 2018;12:CD012378. https://doi.org/10.1002/14651858.CD012378.pub2 